\title{
Quantum Correction to Generalized $T$ Dualities
}

\author{
Riccardo Borsato ${ }^{*}$ \\ Instituto Galego de Física de Altas Enerxías (IGFAE), Universidade de Santiago de Compostela, 15782 Santiago de Compostela, Spain \\ Linus Wulff $\oplus^{\dagger}$ \\ Department of Theoretical Physics and Astrophysics, Masaryk University, 61137 Brno, Czech Republic
}

(Received 22 July 2020; accepted 29 September 2020; published 13 November 2020)

\begin{abstract}
Poisson-Lie duality is a generalization of Abelian and non-Abelian $T$ duality, and it can be viewed as a map between solutions of the low-energy effective equations of string theory, i.e., at the (super) gravity level. We show that this fact extends to the next order in $\alpha^{\prime}$ (two loops in $\sigma$-model perturbation theory) provided that the map is corrected. The $\alpha^{\prime}$ correction to the map is induced by the anomalous Lorentz transformations of the fields that are necessary to go from a doubled $O(D, D)$-covariant formulation to the usual (super)gravity description.
\end{abstract}

DOI: 10.1103/PhysRevLett.125.201603

Introduction.-The notion of $T$ duality [1,2] is central in string theory. It says that a closed string on a background with Abelian isometries has another description as a string on a dual background. In the simplest case of $T$ duality on a circle, the duality acts by inverting the radius of the circle. More generally, backgrounds may have non-Abelian isometry groups, and at least at the classical level there is indeed a generalization to a non-Abelian version of $T$ duality [3]. Unlike in the Abelian case, non-Abelian $T$ duality (NATD) does not generically preserve the isometries of the background, and it is therefore not obvious how to invert the transformation. This problem was overcome by Klimčík and Ševera in $[4,5]$. They realized that the map can be made invertible by relaxing the notion of isometry. One requires the background to have instead so-called Poisson-Lie (PL) symmetry, namely to possess vector fields $v_{i}$, with $\left[v_{i}, v_{j}\right]=-f_{i j}{ }^{k} v_{k}$, under which the metric and $B$ field of the $\sigma$-model transform as

$$
\mathcal{L}_{v_{i}} M_{m n}=-\tilde{f}^{j k}{ }_{i} v_{j}{ }^{p} v_{k}{ }^{q} M_{m p} M_{q n},
$$

where $M_{m n}=G_{m n}-B_{m n}$ and $\tilde{f}^{j k}{ }_{i}$ are structure constants of a dual Lie algebra. This more general notion of symmetry allows to define a dual background (see below). This construction became known as "Poisson-Lie $T$ duality" since the group structure underlying it is that of a PL group. The $\sigma$ models on the original and dual backgrounds are classically equivalent being related by a canonical

Published by the American Physical Society under the terms of the Creative Commons Attribution 4.0 International license. Further distribution of this work must maintain attribution to the author(s) and the published article's title, journal citation, and DOI. Funded by SCOAP ${ }^{3}$. transformation [6]. When the dual structure constants $\tilde{f}$ vanish, $v_{i}$ generate standard isometries, and one recovers (N)ATD.

At the world sheet quantum level, i.e., including string $\alpha^{\prime}$ corrections, things are more subtle. While Abelian $T$ duality remains a symmetry of the world sheet conformal field theory to all orders in $\alpha^{\prime}$, it was quickly realized that NATD cannot be a symmetry at the quantum level [7]. At best it can map one world sheet conformal field theory to another-inequivalent-one. It can therefore be used to generate new string backgrounds from old ones. Except for an anomaly when dualizing nonunimodular groups [8,9], this has been shown to work to zeroth order in $\alpha^{\prime}$, i.e., at the low-energy (supergravity) level of the string effective equations, which corresponds to one loop order in $\sigma$-model perturbation theory. Similar results are known for PL duality, see, e.g., [10,11]. It has been a long-standing problem whether PL and NATD can be extended beyond this lowest order.

Here, we show that PL duality can be extended to order $\alpha^{\prime}$, i.e., two loops in the $\sigma$-model perturbation theory, provided that the map is corrected. A special case of our results gives the corrections to NATD. When specifying to the Abelian case we recover the results of [12].

To find this correction we exploit a powerful formulation of the string effective equations inspired from double field theory (DFT). It has long been known that the bosonic string compactified on a $d$ torus has an $O(d, d) T$ duality symmetry [13]. DFT is a field theory where this symmetry is made manifest form the start [14-18] and is therefore well suited to working with $T$ duality. This is achieved by doubling the dimension of the physical manifold, and by imposing a "section condition" which effectively eliminates the dependence on half of the coordinates, giving the correct dimension in the end $(D=26$ for the bosonic string 
and $D=10$ for the superstring). Here, we always work with the standard choice of section, so that the background depends only on the physical coordinates. In this formulation it is rather the dimension of the tangent space that is doubled, and we have two copies of the Lorentz group instead of one [19]. The standard Lorentz group is the diagonal of the doubled one, and under this breaking the equations of DFT reduce to the standard string effective equations, at lowest order in $\alpha^{\prime}$. A crucial point is that at the quantum level it is impossible to preserve both the $O(D, D)$ and the Lorentz covariance of the fields [20-23]. If we insist on fields which transform nicely under $T$ duality and $O(D, D)$, they must transform noncovariantly under Lorentz transformations [24] (see [25] for another manifestation of this fact). The fact that the Lorentz transformation of the fields receives corrections at order $\alpha^{\prime}$ makes the discussion of the Lorentz invariance of the theory nontrivial. But this can be turned into a virtue rather than a shortcoming. In fact the $\alpha^{\prime}$ correction to the Lorentz transformation fixes the correction to the DFT action [24]. Remarkably, this $\alpha^{\prime}$-corrected $O(D, D)$-covariant action correctly reproduces the $\alpha^{\prime}$ corrections to the bosonic and heterotic string effective actions [24].

Our strategy is to use the rewriting of PL duality in the doubled language, where it takes a natural form, see, e.g., [26-30]. The basic fields of the formulation we use, the "generalized fluxes," turn out to be invariant under PL duality. Since the string effective equations, including the first $\alpha^{\prime}$ correction, can be written in terms of the generalized fluxes [24,31], at least to this order PL duality maps solutions of the doubled equations to solutions. At the standard (super)gravity level there are in fact explicit corrections to the PL duality rules. They arise from the noncovariance of the doubled fields under the double Lorentz transformation needed to gauge fix down to the diagonal subgroup and to go to the standard (nondoubled) description. See Fig. 1 for a summary. This strategy was used in [32] to find the $\alpha^{\prime}$ correction to the "homogeneous Yang-Baxter deformations" (related to NATD $[33,34]$ ) and

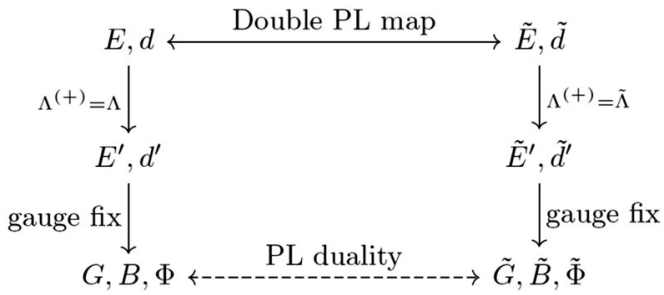

FIG. 1. Starting with the PL duality map for the doubled fields $(E, d)$, the map for the standard (super)gravity fields $(G, B, \Phi)$ is obtained after a double Lorentz transformation $\left(\Lambda^{(+)}, \Lambda^{(-)}\right)=$ $(\Lambda, 1)$ to set $e^{(+)}=e^{(-)}$, thus breaking the double Lorentz group down to its diagonal subgroup. The $\alpha^{\prime}$ corrections to the PL duality map follow from the anomalous Lorentz transformations of the fields. it works for any $O(D, D)$ transformation leaving the generalized fluxes invariant.

Poisson-Lie duality.-In PL duality, $f_{i j}{ }^{k}$ and $\tilde{f}^{i j}{ }_{k}$ are interpreted as structure constants of Lie groups denoted by $G$ and $\tilde{G}$. These are combined into a "Drinfel'd double" $\mathcal{D}$ whose Lie algebra is generated by $T_{I}=\left(T_{i}, \tilde{T}^{i}\right)$, where $T_{i}$ are generators of $\operatorname{Lie}(G)$, and $\tilde{T}^{i}$ of $\operatorname{Lie}(\tilde{G})$. Obviously $\operatorname{Lie}(G)$ and $\operatorname{Lie}(\tilde{G})$ are subalgebras of $\mathcal{D}$ but there are also mixed commutation relations

$$
\begin{aligned}
& {\left[T_{i}, T_{j}\right]=f_{i j}{ }^{k} T_{k}, \quad\left[\tilde{T}^{i}, \tilde{T}^{j}\right]=\tilde{f}^{i j}{ }_{k} \tilde{T}^{k},} \\
& {\left[T_{i}, \tilde{T}^{j}\right]=\tilde{f}^{j k}{ }_{i} T_{k}-f_{i k}{ }^{j} \tilde{T}^{k} .}
\end{aligned}
$$

Importantly, $\mathcal{D}$ is endowed with the invariant symmetric bilinear form $\left\langle T_{I}, T_{J}\right\rangle$ defined by

$$
\left\langle T_{i}, T_{j}\right\rangle=\left\langle\tilde{T}^{i}, \tilde{T}^{j}\right\rangle=0, \quad\left\langle T_{i}, \tilde{T}^{j}\right\rangle=\delta_{i}^{j}
$$

Having introduced $\mathcal{D}$ we can now present PL duality as an invertible map between an "original" background (specified by a metric $G_{m n}$, a Kalb-Ramond field $B_{m n}$, and a dilaton $\Phi$ ) and another "dual" background (with fields $\tilde{G}_{m n}, \tilde{B}_{m n}$, and $\tilde{\Phi}$ ). We split the coordinates of the original background as $x^{m}=\left(y^{\sigma}, x^{\mu}\right)$, where $y^{\sigma}$ are coordinates on the group $G$ to be dualized, and $x^{\mu}$ are coordinates that play the role of spectators under the dualization. Similarly, for the dual background we have $\tilde{x}^{m}=\left(\tilde{y}^{\sigma}, x^{\mu}\right)$ with $\tilde{y}^{\sigma}$ coordinates on $\tilde{G}$. The $y$ and $\tilde{y}$ dependence is in fact encoded in the group elements $g(y) \in$ $G$ and $\tilde{g}(\tilde{y}) \in \tilde{G}$ featuring below. To present the map between the original and dual backgrounds we first need the fact that the condition (1) implies that $M_{m n} \equiv G_{m n}$ $B_{m n}$ is of the form

$$
M=U \dot{M}(1+\Pi \dot{M})^{-1} U^{T},
$$

where we suppressed matrix indices for readability. The matrix $U_{m}{ }^{r}$ depends only on $y$ and it is of block form with nonvanishing components $U_{\mu}{ }^{\nu}=\delta_{\mu}{ }^{\nu}$ and $U_{\sigma}{ }^{i}=u_{\sigma}{ }^{i}$, the latter being the components of the Maurer-Cartan form $u=$ $g^{-1} v g=g^{-1} d g=d y^{\sigma} u_{\sigma}{ }^{i} T_{i}$ [35]. The matrix $\Pi^{r s}$ depends only on $y$ and its only nontrivial components are

$$
\Pi^{i j}=\left\langle A d_{g}^{-1} \circ \mathrm{P} \circ A d_{g} \tilde{T}^{i}, \tilde{T}^{j}\right\rangle,
$$

where $A d_{g} X=g X g^{-1}$ and $\mathrm{P}$ is the projector on $\operatorname{Lie}(G)$. Notice that in general $\Pi \neq 0$ thanks to the mixed commutation relation of $\mathcal{D}$ if $\tilde{f}^{i j}{ }_{k} \neq 0$. The map between $M_{m n}$ and $\tilde{M}_{m n}$ is achieved by relating both of them to $\dot{M}_{r s}$, a matrix depending only on spectators $x^{\mu}$ and on which no other condition is imposed [36]. The dual background $\tilde{M}_{m n}$ is obtained by [37]

$$
\tilde{M}=\tilde{U}[(\dot{M}+\tilde{\Pi}) P+\bar{P}]^{-1}(\dot{M} \bar{P}+P) \tilde{U}^{T},
$$


where $\tilde{U}_{\mu}{ }^{\nu}=\delta_{\mu}{ }^{\nu}, \tilde{U}_{\sigma}{ }^{i}=\tilde{u}_{\sigma j} \delta^{j i}$ and $P, \bar{P}$ project on indices $i, j$ and $\mu, \nu$, respectively. As previously $\tilde{u}=\tilde{g}^{-1} d \tilde{g}=$ $d \tilde{y}^{\sigma} \tilde{u}_{\sigma i} \tilde{T}^{i}$ is a Maurer-Cartan form, and now $\tilde{\Pi}_{i j}=$ $\left\langle A d_{\tilde{g}}^{-1} \circ \tilde{\mathrm{P}} \circ A d_{\tilde{g}} T_{i}, T_{j}\right\rangle$, where $\tilde{\mathrm{P}}$ projects on $\operatorname{Lie}(\tilde{G})$. Finally, the dilatons of the two backgrounds are related by [38]

$$
\exp (-2 \Phi) \frac{(\operatorname{det} G)^{1 / 2}}{\operatorname{det} u}=\exp (-2 \tilde{\Phi}) \frac{(\operatorname{det} \tilde{G})^{1 / 2}}{\operatorname{det} \tilde{u}}
$$

Taking $\tilde{G}$ Abelian $\left(\tilde{f}^{i j}{ }_{k}=0\right)$ implies $\Pi=0$ and Eq. (4) simplifies to $M=U \dot{M} U^{T}$, encoding the usual consequences of having isometries for $M$. Then parametrizing the Abelian group as $\tilde{g}=\exp \left(\tilde{y}_{i} \tilde{T}^{i}\right)$ with $\tilde{y}_{i}=\tilde{y}^{\sigma} \delta_{\sigma i}$ it follows that $\tilde{u}_{\sigma i}=\delta_{\sigma i}, \tilde{\Pi}_{i j}=\tilde{y}_{k} f_{i j}{ }^{k}$, and from (6) and (7) we recover the rules of NATD in the presence of spectators [39]. Even simpler is the case when also $G$ is Abelian $\left(f_{i j}{ }^{k}=0\right)$ so that $M$ is invariant under $\operatorname{dim}(G) U(1)$ isometries. Then also $\tilde{\Pi}=0$ and Eq. (6) implements dim $(G)$ factorized $T$ dualities, reducing to the celebrated Buscher rules when only one isometry is dualized.

Double formulation.-The nonlinear maps in (4) and (6) admit a much simpler and linear formulation in the doubled language, where one works with matrices $\mathcal{O}_{M}{ }^{N}$ of dimension $2 D \times 2 D$. These are elements of the group $O(D, D)$, meaning that $\mathcal{O}_{M}{ }^{P} \mathcal{O}_{N} Q_{\eta_{P Q}}=\eta_{M N}$ where

$$
\eta_{M N}=\left(\begin{array}{cc}
0 & \delta^{m}{ }_{n} \\
\delta_{m}{ }^{n} & 0
\end{array}\right) .
$$

In fact let us construct the (inverse) "generalized vielbein" which we parametrize as

$$
E_{A}{ }^{M}=\frac{1}{\sqrt{2}}\left(\begin{array}{cc}
e^{(+) a n} M_{n m} & e^{(+) a m} \\
-e_{a}^{(-) n} M_{n m} & e_{a}^{(-) m}
\end{array}\right),
$$

where $A$ is a flat index and $M$ curved. We use similar parametrizations for $\tilde{E}_{A}{ }^{M}$ and $\dot{E}_{A}{ }^{R}$, adding tildes and dots. Above, $e^{( \pm)}$are two possible vielbeins for the metric $G_{m n}$. They are not necessarily equal and in general they are related by a nontrivial Lorentz transformation. Each of them transform under only one of the two copies of the Lorentz group [distinguished by the (+) and (-)] arising in the doubled formulation. The generalized vielbein is one of the main ingredients of the "framelike formulation" of DFT, and it will be important for our derivation of the "unimodularity condition" (18) and the $\alpha^{\prime}$ corrections to PL duality. It is straightforward to check that the relations (4) and (6) are equivalent to the relations

$$
E=\dot{E}(1+\Pi) \mathcal{U}, \quad \tilde{E}=\dot{E}(1+\tilde{\Pi}) \tilde{\mathcal{U}},
$$

where we suppressed indices. In our notation all dotted quantities only depend on $x^{\mu}$. The nonvanishing components of $\Pi_{R} S$ and $\tilde{\Pi}_{R}{ }^{S}$ are again only $\Pi^{i j}$ and $\tilde{\Pi}_{i j}$ and the antisymmetry properties $\Pi^{i j}=-\Pi^{j i}$ and $\tilde{\Pi}_{i j}=$ $-\tilde{\Pi}_{j i}$ imply that $(1+\Pi),(1+\tilde{\Pi})$ are elements of $O(D, D)$. The matrices $\mathcal{U}, \tilde{\mathcal{U}}$ are also elements of $O(D, D)$ with $\mathcal{U}_{i}{ }^{\sigma}=u_{i}{ }^{\sigma}, \mathcal{U}^{i}{ }_{\sigma}=u_{\sigma}{ }^{i}, \tilde{\mathcal{U}}^{i \sigma}=\tilde{u}^{i \sigma}, \tilde{\mathcal{U}}_{i \sigma}=\tilde{u}_{\sigma i}, \mathcal{U}_{\mu}{ }^{\nu}=\mathcal{U}^{\mu}{ }_{\nu}=$ $\tilde{\mathcal{U}}_{\mu}{ }^{\nu}=\tilde{\mathcal{U}}^{\mu}{ }_{\nu}{ }=\delta_{\mu}{ }^{\nu}$. We are using a notation so that $u_{i}^{\sigma}$ and $\tilde{u}^{i \sigma}$ are the inverses of $u_{\sigma}{ }^{i}$ and $\tilde{u}_{\sigma i}$, respectively. To match (4) and (6) with (10) one finds that the (+) and (-) vielbeins must transform differently

$$
\begin{aligned}
& e_{a}^{( \pm) m}=\dot{e}_{a}^{( \pm) s} O_{( \pm) s}{ }^{r}\left(U^{-1}\right)_{r}{ }^{m}, \\
& \tilde{e}_{a}^{( \pm) m}=\dot{e}_{a}^{( \pm) s} \tilde{O}_{( \pm) s}{ }^{r}\left(\tilde{U}^{-1}\right)_{r}{ }^{m},
\end{aligned}
$$

where

$O_{(+)}=1+\dot{M} \Pi, \quad \tilde{O}_{(+)}=\bar{P}+(\tilde{\Pi}+\dot{M}) P$,

$O_{(-)}=1-\dot{M}^{T} \Pi, \quad \tilde{O}_{(-)}=\bar{P}+\left(\tilde{\Pi}-\dot{M}^{T}\right) P$.

In both cases the $(+)$ and $(-)$ vielbeins are then related by Lorentz transformations as $e_{a}^{(-) m}=\Lambda_{a}^{b} e_{b}^{(+) m}$ and $\tilde{e}_{a}^{(-) m}=$ $\tilde{\Lambda}_{a}^{b} \tilde{e}_{b}^{(+) m}$ where

$$
\Lambda=\dot{e}^{-1} O_{(-)} O_{(+)}^{-1} \dot{e}, \quad \tilde{\Lambda}=\dot{e}^{-1} \tilde{O}_{(-)} \tilde{O}_{(+)}^{-1} \dot{e},
$$

if we fix $\dot{e}=\dot{e}^{(+)}=\dot{e}^{(-)}$. Finally, the transformation (7) is translated into

$$
d+\frac{1}{2} \log \operatorname{det} u=\dot{d}=\tilde{d}+\frac{1}{2} \log \operatorname{det} \tilde{u},
$$

where $d, \dot{d}, \tilde{d}$ are called "generalized dilatons" and are parametrized as in $d=\Phi-\frac{1}{4} \log \operatorname{det} G$ [41].

$P L$ duality as a map between string backgrounds.-The double formulation is very useful because in this language it is very simple to prove that the PL duality transformation is a solution generating technique in string theory, at least to leading and subleading order in the $\alpha^{\prime}$ expansion. From $E_{A}{ }^{M}$ and $d$ one can construct the generalized fluxes

$$
\begin{aligned}
\mathcal{F}_{A B C} & =3 E_{[A}{ }^{M} \partial_{M} E_{B}{ }^{N} E_{C] N}, \\
\mathcal{F}_{A} & =E^{B M} \partial_{M} E_{B}{ }^{N} E_{A N}+2 E_{A}{ }^{M} \partial_{M} d,
\end{aligned}
$$

that are the dynamical fields of the framelike formulation of DFT. In fact the DFT equations of motion can be written only in terms of the above fluxes and their flat derivatives $\partial_{A} \mathcal{F}=E_{A}{ }^{M} \partial_{M} \mathcal{F}$, both at leading and subleading order in the $\alpha^{\prime}$ expansion [31]. Under the transformation (10) we have

$$
\begin{aligned}
\mathcal{F}_{A B C}= & 3 \dot{E}_{[A}{ }^{[A} \partial_{\mu} \dot{E}_{B}{ }^{N} \dot{E}_{C] N} \\
& +3 \dot{E}_{[A}{ }^{i} \dot{E}_{B}{ }^{j} \dot{E}_{C] k} f_{i j}{ }^{k}+3 \dot{E}_{[A}{ }^{i} \dot{E}_{B j} \dot{E}_{C] k} \tilde{f}^{j k}{ }_{i},
\end{aligned}
$$




$$
\begin{aligned}
\mathcal{F}_{A}= & \dot{E}^{B \mu} \partial_{\mu} \dot{E}_{B}{ }^{N} \dot{E}_{A N}+2 \dot{E}_{A}{ }^{\mu} \partial_{\mu} \dot{d} \\
& +\dot{E}_{A}{ }^{i} f_{i j}{ }^{j}-\dot{E}_{A i}\left(\tilde{f}^{i j}{ }_{j}+f_{j k}{ }^{i} \Pi^{j k}\right) .
\end{aligned}
$$

For the reader's convenience we give the details of the computation in the Supplemental Material [37]. The results for the dual background are analogous upon exchanging tilded and untilded quantities, and appropriately raising or lowering $i, j, k$ indices. Because of the symmetry of (16) under this transformation, it immediately follows that $\mathcal{F}_{A B C}=\tilde{\mathcal{F}}_{A B C}$. Equation (17) instead is not symmetric under this transformation, but it becomes symmetric if we impose the tracelessness of the structure constants

$$
f_{i j}{ }^{j}=0, \quad \tilde{f}^{i j}{ }_{j}=0,
$$

as detailed in the Supplemental Material. When this "unimodularity condition" holds we have simply

$$
\mathcal{F}_{A}=\dot{E}^{B \mu} \partial_{\mu} \dot{E}_{B}{ }^{N} \dot{E}_{A N}+2 \dot{E}_{A}{ }^{\mu} \partial_{\mu} \dot{d}
$$

and $\mathcal{F}_{A}=\tilde{\mathcal{F}}_{A}$ immediately follows. Notice that not only both fluxes but also their flat derivatives are invariant under the PL transformation. In fact, since they only depend on spectators $x^{\mu}$ it follows that $E_{A}{ }^{M} \partial_{M} \mathcal{F}=E_{A}{ }^{\mu} \partial_{\mu} \mathcal{F}=\tilde{E}_{A}{ }^{\mu} \partial_{\mu} \tilde{\mathcal{F}}=\tilde{E}_{A}{ }^{M} \tilde{\partial}_{M} \tilde{\mathcal{F}}$.

If we start from a string background, or in other words given a model with $E_{A}{ }^{M}$ and $d$ of the PL form (10) and (14) that satisfies the doubled equations of motion to zeroth and first order in $\alpha^{\prime}$, we conclude that the dual model given by $\tilde{E}_{A}{ }^{M}$ and $\tilde{d}$ also satisfies the same equations, at least when (18) holds. This observation extends to higher orders under the assumption that there exists a formulation of the string effective action in terms of the generalized fluxes and their flat derivatives [31] also at higher orders in $\alpha^{\prime}$. This in turn should be true as long as it is possible to make diffeomorphisms, $B$-field gauge transformations, and $O(D, D)$ symmetry manifest.

This is a proof that PL duality is a solution generating technique in string theory at least when both structure constants are traceless, as found already to lowest order in [42]. When $\tilde{G}$ is Abelian this condition reduces to the unimodularity condition for NATD [8,9].

$\alpha^{\prime}$ corrections.-So far, we have shown that in the doubled formulation the PL duality transformation works and remains uncorrected at least to order $\alpha^{\prime}$. Note that the assumption is that the DFT equations are satisfied without the need of correcting the $O(D, D)$ form (10) of the PL transformation, and therefore only $\dot{M}$ and $\dot{d}$ in (10) and (14) can depend on $\alpha^{\prime}$.

The description of the two models in terms of standard (i.e., nondoubled) fields $(G, B, \Phi)$ and $(\tilde{G}, \tilde{B}, \tilde{\Phi})$ is different, and the PL duality transformation between these does receive $\alpha^{\prime}$ corrections. The reason is that when going from a doubled to a standard (super)gravity formulation we must first perform a double Lorentz transformation to set the two vielbeins $e^{(+)}$and $e^{(-)}$equal [24]. At order $\alpha^{\prime}$ the fields of the doubled formulation transform noncovariantly under local Lorentz transformations, and this induces extra $\alpha^{\prime}$ corrections also for the standard fields. The situation is illustrated in Fig. 1. Because of the noncovariance even under the diagonal of the double Lorentz group, we say that the reduction from the doubled to the standard formulation picks a specific noncovariant "scheme," which we call the scheme of DFT. To translate our results into the covariant schemes of [43-46] one must implement $\alpha^{\prime}$-dependent field redefinitions. We provide a dictionary [47] in the Supplemental Material [37].

The first $\alpha^{\prime}$ correction to $M_{m n}$ induced by the compensating double Lorentz transformation with $\Lambda^{(+)}$and $\Lambda^{(-)}$ is [48]

$$
a \Delta_{\Lambda^{(-)}}^{(-)} M_{n m}^{(\mathrm{DFT})}+b \Delta_{\Lambda^{(+)}}^{(+)} M_{m n}^{(\mathrm{DFT})}
$$

where $a=b=-\alpha^{\prime}$ for the bosonic string and $a=-\alpha, b=$ 0 for the heterotic string (and $a=b=0$ for type II). The finite form of the anomalous transformations is [37]

$$
\begin{aligned}
\Delta_{\Lambda}^{( \pm)} M_{m n}^{(\mathrm{DFT})}= & \frac{1}{2} \operatorname{tr}\left(\partial_{m} \Lambda \Lambda^{-1} \omega_{n}^{( \pm)}\right)-B_{m n}^{\mathrm{WZW},(\Lambda)} \\
& +\frac{1}{4} \operatorname{tr}\left(\partial_{m} \Lambda \Lambda^{-1} \partial_{n} \Lambda \Lambda^{-1}\right)
\end{aligned}
$$

where $\omega_{m a}^{( \pm) b}=\omega_{m a}{ }^{b} \pm \frac{1}{2} H_{m a}{ }^{b}$ and $\omega$ is the spin connection for the vielbein $e$ after the diagonal gauge fixing. The WZW-like contribution to $B$ is defined by

$$
d B^{\mathrm{WZW},(\Lambda)}=-\frac{1}{12} \operatorname{tr}\left(d \Lambda \Lambda^{-1} d \Lambda \Lambda^{-1} d \Lambda \Lambda^{-1}\right) .
$$

The $\alpha^{\prime}$ corrections to the original model can be obtained, for example, after choosing $e=e^{(-)}$and doing the double Lorentz transformation on $e^{( \pm)}$to achieve the diagonal gauge with $\left(\Lambda^{(+)}, \Lambda^{(-)}\right)=(\Lambda, 1)$ and $\Lambda$ given in (13) [49]. Then the correction is

$$
\begin{aligned}
\Delta M^{(\mathrm{DFT})}= & b \Delta_{\Lambda}^{(+)} M^{(\mathrm{DFT})} \\
& +\alpha^{\prime} U(1+\dot{M} \Pi)^{-1} \Delta \dot{M}(1+\Pi \dot{M})^{-1} U^{T},
\end{aligned}
$$

where the second term comes when expanding (4) with the $\alpha^{\prime}$ corrections $\dot{M} \rightarrow \dot{M}+\alpha^{\prime} \Delta \dot{M}$. Notice that for the heterotic string $(b=0)$ the PL map is uncorrected in the DFT scheme in the gauge $e=e^{(-)}$[50]. For the dual background the same reasoning applies, and choosing $\tilde{e}=\tilde{e}^{(-)}$ 


$$
\begin{aligned}
\Delta \tilde{M}^{(\mathrm{DFT})}= & b \Delta_{\tilde{\Lambda}}^{(+)} \tilde{M}^{(\mathrm{DFT})} \\
& +\alpha^{\prime} \tilde{U}(\dot{M} P+\tilde{\Pi}+\bar{P})^{-1} \Delta \dot{M}\left(-P \tilde{U}^{-1} \tilde{M}+\bar{P} \tilde{U}^{T}\right) .
\end{aligned}
$$

The transformation of the dilatons follows from the fact that the generalized dilaton (14) is not anomalous under Lorentz [24] and that the parametrization in terms of standard metric and dilaton holds to $\alpha^{\prime}$ order. Then

$$
\begin{aligned}
& \Delta \Phi^{(\mathrm{DFT})}=\alpha^{\prime} \Delta \dot{d}+\frac{1}{4} G^{m n} \Delta G_{m n}^{(\mathrm{DFT})}, \\
& \Delta \tilde{\Phi}^{(\mathrm{DFT})}=\alpha^{\prime} \Delta \dot{d}+\frac{1}{4} \tilde{G}^{m n} \Delta \tilde{G}_{m n}^{(\mathrm{DFT})},
\end{aligned}
$$

where we allowed an $\alpha^{\prime}$ correction $\dot{d} \rightarrow \dot{d}+\alpha^{\prime} \Delta \dot{d}$.

We refer to the Supplemental Material for an example of a computation of such $\alpha^{\prime}$ corrections, and for Refs. [51,52]. When specifying the map to a single $U(1) T$ duality, Eqs. (24) and (25) reproduce the rules written in [12] by Kaloper and Meissner, as proved in [32].

Conclusions. - In this Letter, we employed the framelike formulation of DFT to show that [when the conditions in (18) hold] PL duality is a map between solutions of the lowenergy effective string equations at least to first order in $\alpha^{\prime}$ and quite possibly to all orders. We did this for a twoparameter family of theories interpolating between the bosonic and the heterotic string (when the gauge fields and fermions of the latter are set to zero). It would be interesting to generalize these results to the case in which $G$, for example, is replaced by the coset $G / H$.

The importance of Eqs. (23), (24), and (25) is twofold. First, they provide the necessary quantum corrections to the PL duality transformation rules in order to extend the map to order $\alpha^{\prime}$. Second, they imply that the form of the $\alpha^{\prime}$ corrections of backgrounds admitting PL symmetry is strongly constrained by the PL symmetry itself [53]. In particular, Eqs. (23) and (25) can be interpreted as an efficient way to compute $\alpha^{\prime}$ corrections for PL symmetric backgrounds, since the only unknowns are $\Delta \dot{M}$ and $\Delta \dot{d}$, and they can be found by imposing the order $\alpha^{\prime}$ equations of motion. This is much simpler than trying to compute the corrections directly for $M$ and $\Phi$. It would be interesting to see if, when considering nonconformal $\sigma$ models, the $\alpha^{\prime}$ corrections that we find preserve the form of the $\beta$ functions.

The work of R. B. is supported by the fellowship of "la Caixa Foundation" (ID 100010434) with code LCF/BQ/ PI19/11690019, by Agencia Estatal de InvestigaciónSpain (FPA2017-84436-P and Unidad de Excelencia María de Maetzu MDM-2016-0692), by Xunta de Galicia-Consellería de Educación (Centro singular de investigación de Galicia accreditation 2019-2022, ED431C-2017/07 and ED431G2019/05), and by
FEDER. The work of L.W. is supported by the grant "Integrable Deformations" (GA20-04800S) from the Czech Science Foundation (GACR).

Note added.-When this work was being written up we learned of the closely related independent work [54], and shortly after of [55].

*riccardo.borsato@usc.es †wulff@physics.muni.cz

[1] T. H. Buscher, Phys. Lett. B 194, 59 (1987).

[2] T. Buscher, Phys. Lett. B 201, 466 (1988).

[3] X. C. de la Ossa and F. Quevedo, Nucl. Phys. B403, 377 (1993).

[4] C. Klimcik and P. Severa, Phys. Lett. B 351, 455 (1995).

[5] C. Klimcik and P. Severa, Phys. Lett. B 372, 65 (1996).

[6] K. Sfetsos, Nucl. Phys. B517, 549 (1998).

[7] A. Giveon and M. Rocek, Nucl. Phys. B421, 173 (1994).

[8] E. Alvarez, L. Alvarez-Gaume, and Y. Lozano, Nucl. Phys. B424, 155 (1994).

[9] S. Elitzur, A. Giveon, E. Rabinovici, A. Schwimmer, and G. Veneziano, Nucl. Phys. B435, 147 (1995).

[10] A. Alekseev, C. Klimcik, and A. A. Tseytlin, Nucl. Phys. B458, 430 (1996).

[11] E. Tyurin and R. von Unge, Phys. Lett. B 382, 233 (1996).

[12] N. Kaloper and K. A. Meissner, Phys. Rev. D 56, 7940 (1997).

[13] A. Giveon, M. Porrati, and E. Rabinovici, Phys. Rep. 244, 77 (1994).

[14] W. Siegel, Phys. Rev. D 47, 5453 (1993).

[15] W. Siegel, Phys. Rev. D 48, 2826 (1993).

[16] G. Aldazabal, D. Marques, and C. Nunez, Classical Quantum Gravity 30, 163001 (2013).

[17] D. S. Berman and D. C. Thompson, Phys. Rep. 566, 1 (2015).

[18] O. Hohm, D. Lüst, and B. Zwiebach, Fortschr. Phys. 61, 926 (2013).

[19] O. Hohm and S. K. Kwak, J. Phys. A 44, 085404 (2011).

[20] O. Hohm and B. Zwiebach, J. High Energy Phys. 05 (2012) 126.

[21] O. Hohm, W. Siegel, and B. Zwiebach, J. High Energy Phys. 02 (2014) 065.

[22] O. Hohm and B. Zwiebach, J. High Energy Phys. 11 (2014) 075.

[23] O. Hohm and B. Zwiebach, J. High Energy Phys. 01 (2015) 012.

[24] D. Marqués and C. A. Nuñez, J. High Energy Phys. 10 (2015) 084.

[25] C. Eloy, O. Hohm, and H. Samtleben, Phys. Rev. Lett. 124, 091601 (2020).

[26] F. Hassler, Phys. Lett. B 807, 135455 (2020).

[27] B. Jurco and J. Vysoky, J. Geom. Phys. 130, 1 (2018).

[28] S. Demulder, F. Hassler, and D. C. Thompson, J. High Energy Phys. 02 (2019) 189.

[29] Y. Sakatani, Prog. Theor. Exp. Phys. 2019, 073 B04 (2019).

[30] A. Catal-Ozer, J. High Energy Phys. 08 (2019) 115.

[31] W. H. Baron, J. J. Fernandez-Melgarejo, D. Marques, and C. Nunez, J. High Energy Phys. 04 (2017) 078. 
[32] R. Borsato, A. V. López, and L. Wulff, J. High Energy Phys. 07 (2020) 103.

[33] B. Hoare and A. A. Tseytlin, J. Phys. A 49, 494001 (2016).

[34] R. Borsato and L. Wulff, Phys. Rev. Lett. 117, 251602 (2016).

[35] We will use indices $r, s$ where $r=(i, \mu)$ to distinguish them from indices $m, n$ where $m=(\sigma, \mu)$.

[36] We obviously have to assume invertibility of the inverse matrices in (4) and (6).

[37] See the Supplemental Material at http://link.aps.org/ supplemental/10.1103/PhysRevLett.125.201603 for a rewriting of this formula more familiar to the usual form of the $(\mathrm{N})$ ATD rules. In the case of no spectators (and assuming invertibility of $\dot{M}$ ) Eq. (4) reduces to $M=$ $u\left(\dot{M}^{-1}+\Pi\right)^{-1} u^{T}$ and Eq. (6) to $\tilde{M}=\tilde{u}(\dot{M}+\tilde{\Pi})^{-1} \tilde{u}^{T}$, which are more symmetric and more familiar in the PL duality literature. Also, we review how to obtain the finite form of the transformation used in (21) from the infinitesimal one of [24], as explained in [32].

[38] R. Von Unge, J. High Energy Phys. 07 (2002) 014.

[39] Compared to [40] here $\tilde{y}_{i}=-\nu_{i}$ and we swap $\tilde{e}^{( \pm)}$ appearing later.

[40] R. Borsato and L. Wulff, J. High Energy Phys. 08 (2018) 027.

[41] Because $\dot{d}$ depends only on $x^{\mu}$ it follows that $\partial_{\sigma} \Phi=$ $\frac{1}{2} \partial_{\sigma} \log \operatorname{det} M-\partial_{\sigma} \log \operatorname{det} u$ and similarly for $\tilde{\Phi}$.
[42] A. Bossard and N. Mohammedi, Nucl. Phys. B619, 128 (2001).

[43] E. Bergshoeff and M. de Roo, Phys. Lett. B 218, 210 (1989).

[44] E. A. Bergshoeff and M. de Roo, Nucl. Phys. B328, 439 (1989).

[45] R. R. Metsaev and A. A. Tseytlin, Nucl. Phys. B293, 385 (1987).

[46] C. M. Hull and P. K. Townsend, Nucl. Phys. B301, 197 (1988).

[47] R. Borsato and L. Wulff, J. High Energy Phys. 03 (2020) 126.

[48] Notice the first transposition.

[49] Notice that it is not possible to set directly $e^{(+)}=e^{(-)}$ without the help of a Lorentz transformation, as this would be incompatible with the assumption that $\dot{e}^{( \pm)}$depend only on $x^{\mu}$. Therefore we used the gauge $\dot{e}=\dot{e}^{(+)}=\dot{e}^{(-)}$.

[50] Alternatively, one may choose $e=e^{(+)}$and the anomalous terms would be $a \Delta_{\Lambda^{-1}}^{(-)} M_{n m}^{(\mathrm{DFT})}$.

[51] A. Eghbali, Phys. Rev. D 99, 026001 (2019).

[52] A. Eghbali, R. Naderi, and A. Rezaei-Aghdam, arXiv:2002.00675.

[53] This is under the assumption that the PL symmetry is not anomalous.

[54] F. Hassler and T. Rochais, arXiv:2007.07897.

[55] T. Codina and D. Marques, arXiv:2007.09494. 